\title{
Some Simulation Results and Parameter Analyses of a Generalized Quasi Two-Phase Bulk Mixture Model
}

\author{
Khim B. Khattri ${ }^{1}$, Parameshwari Kattel ${ }^{1,2}$, Bhadra Man Tuladhar ${ }^{1}$ \\ ${ }^{1}$ Department of Mathematics, School of Science, Kathmandu University, Dhulikhel, Kavre, Nepal \\ 2 Department of Mathematics, Tri-chandra Multiple Campus, Tribhuvan University, Kathmandu, Nepal \\ Correspondence to: Khim B. Khattri, Email: khim_khatri@student.ku.edu.np
}

\begin{abstract}
Here, we consider a newly constructed generalized quasi two-phase bulk model for a rapid flow of a debris mixture consisting of viscous fluid and solid particles down a channel. The model is a set of coupled partial differential equations with new mechanical description of generalized bulk and shear viscosities, pressure, velocities and effective friction. The dynamical variables, physical parameters, inertial and dynamical coefficients and drift factors involved in the equations contain certain important physics of mixture flow. So, we analyze the behaviour of some inertial and dynamical coefficients involved in the model. These coefficients strongly depend upon the initial material composition. We also simulate the evolution of the dynamical variables so as to reveal their strong non-linear behaviours. Through simulations, we also analyze the front position of the debris material, bottom-slip velocity and maximum velocity, which show fundamentally different dynamics for varied material compositions, from dilute to dense flows. Generalized mixture viscosity decreases as the flow moves downslope, and the rate at which it decreases depends upon the initial solid-volume fraction. We also compare the effective viscosity and the bulk mixture viscosity with respect to the norm of the strain rate tensors to reveal different non-linear behaviours.
\end{abstract}

Keywords: Drift factors, Inertial and dynamical coefficients, Generalized bulk and mixture model

\section{Introduction}

Debris flows are rapidly moving mixture of poorly sorted sediment and water triggered by some climatic factors, erosion, anthropogenic activities, or inertial accelerations induced by earthquakes or volcanic eruptions, causing a major threat to human life, property, infrastructures and natural environment in most mountainous and hilly regions around [11, 29, 33, 35. In natural debris flows, both solid and fluid phases velocities may differ substantially from each other, essentially affecting flow mechanics [31, 33, 38, 39. Their flow characteristics depend on the sediment composition (volume fraction, size, sorting, etc.) and on the dynamic interaction between the solid and fluid phases [10, 33. The modeling of the debris flow and the phase-interaction are still facing difficulties due to the lack of correct rheology and scales, and the ongoing computational models largely rely on simplifying assumptions [11. Proper understanding of mass flow mechanism and the estimation of their devastating effects are required for the effective mitigation.

There are significant research efforts in modeling the mechanical behaviour of debris materials. Previously, various researchers made different attempts to model debris flows being based on different rheological models: Newtonian models [17, linear and non-linear viscoplastic models [28, dilatant fluid models [2], dispersive or turbulent stress models [1, biviscous modified Bingham model [4, and frictional models [27. Among them, linear (Bingham) or non-linear (Herschel-Bulkey) viscoplastic models are found to be widely used to describe the rehology of laminar debris/mud flows [16]. In the viscoplastic model of Johnson [16], debris flow was simplified as a single phase continuum with Bingham or Coulomb yield strength. However, this model could not describe the interaction between particles and fluid. Dilatant fluid model was proposed by Takahashi [41] being based on Bagnold's research. Theoretically, single-phase flow models have been developed considering either solid or fluid, or a mixture of both during the past decades. Savage and Hutter [40] proposed the depth averaged avalanche model, a pioneer work in modeling effort with the assumptions: (i) incompressible flow, (ii) shallow avalanche piles with small topographic curvatures, (iii) Coulomb sliding with bed friction angle $\delta$, and (iv) Mohr-Coulomb behaviour in the interior with the friction angle $\phi \geq \delta$ along with an ad-hoc assumption reducing the number of Mohr's circles in three dimensional stress states to a single. There were other significant fundamental research activities in the past few decades in the field of debris flow and related gravitational mass flows. To mention a few, single-phase dry granular avalanches [8, 12, 13, 34, 46, single-phase debris flows [2, 3, 28, 32, 42, mixture flows [14, 15, 36, two-fluid debris 
flow [29] and two layered model [7]. These models had to rely on significant limitations and assumptions to reproduce the field or the laboratory data not being able to describe extremely complex fluid-particle interacting flows. Modeling of a debris flow is more challenging than the simple water flow due to its multiphase character and the interaction of the phases. In natural debris flows, the flow regime often changes in space and time; the debris head may behave quite differently than other parts of the debris body, typically, the tail [33].

Pudasaini 33 proposed a dynamical model that accounts for strong interactions between the solid and the fluid phases with full details. This model constitutes the most generalized two-phase avalanches and debris flow model to date that includes important mechanics of two-phase mass flows like, buoyancy, solid-volumefraction-gradient enhanced non-Newtonian viscous stress, virtual mass, and generalized drag. Relaxing one or more of the aforementioned aspects reduces the model into other relatively simpler previous models those exist in the literature for debris flows and avalanches. Worni et al. [45] also emphasized the need to consider the two-phase flow models to account for the evolution and interactions between the phases. Using the model of Pudasaini [33, various numerical experiments in generic topographies related to glacial lake outburst floods (GLOFs) 20], landslide induced tsunami [18, 19, two phase debris flow-obstacle-interactions 21, 22 were performed. The analytical solutions of the convective-diffusive equation as derived from $\mathrm{Pu}-$ dasaini [33] can be found on Pudasaini et al. 37.

Using physically based two-phase mass flow model 33, Pokhrel et al. 30. constructed a bulk model that consists of two dimensional quasi two-phase mass and momentum equations for generalized mixture velocities and pressure for the faster simulations than the two-phase models and for more accurate modeling than the existing effectively single-phase models. The model appears in non-conventional form due to the inertial coefficients, and the complex mixture viscosities. This structure resulted in the emergence of a new and dynamically evolving effective mixture friction coefficient, and general mixture viscosity. Recently, Khattri and Pudasaini [26] presented a novel simulations of generalized quasi two-phase mass flow model to exhibit new non-linear phenomena and mechanical insights of the model alongwith a full discretization of the model for the generalized mixture viscosity, velocity, pressure and effective bulk friction with higher order central differences and donor-cell method. Fundamentally new results for the generalized mixture viscosity, velocity, pressure and effective bulk friction along with the analysis of the performance of the full dimensional evolution of mixture velocities, dynamic pressure and effective viscosity are presented in their work. Khattri and Pudasaini 24] have constructed an extended generalized quasi two-phase mass flow model so as to include the essential physics of two-phase mass flow such as non-Newtonial viscous stress, generalized drag and virtual mass in mixture mass flow. This model has been used to simulate dry, and wet snow avalanches of different water contents and their obstacle interactions in Khattri et al. 25].

In this contribution, we analyze the behaviour of drift factors and inertial coefficients in downslope distance for dilute to dense flows i.e., for different material composition. Following the simulations of Khattri and Pudasaini [26], we also analyze the maximum velocities and front positions of velocities in downslope distance for different solid volume fractions. We also present the time evolution of bottom slip velocities and mixture velocities. We also compare the evolution of effective viscosity [6] and recently constructed generalized mixture viscosity [30]. The main theme of this contribution is to analyze how the velocity drift factors affect in the behaviour of other dynamical variables during the mixture flow along a channel. Computation of the mixture velocities and pressure is not within the scope of this and will appear in future contribution.

\section{Generalized Bulk Mixture Model}

The generalized quasi two-phase mass flow model [30] in vector form can be written as

$$
\operatorname{div} \mathbf{u}_{m}^{\Lambda}=0, \frac{D \mathbf{u}_{m}^{\Lambda}}{D t}=\operatorname{div} \boldsymbol{\sigma}_{m}+\mathbf{f},
$$

where $\frac{D \mathbf{u}_{m}^{\Lambda}}{D t}$ is the material derivative of the mixture velocity vector $\mathbf{u}_{\mathbf{m}}^{\Lambda}=\left(\Lambda_{u} u_{m}, \Lambda_{w} w_{m}\right) ; \Lambda_{u}=$ 
$\frac{1}{\alpha_{s}+\lambda_{u} \alpha_{f}}, \Lambda_{w}=\frac{1}{\alpha_{s}+\lambda_{w} \alpha_{f}}$. The drift factors $\lambda_{u}$, and $\lambda_{w}$ express velocity of one phase to the other phase so that fluid momentum equations in Pudasaini 33. can be expressed in terms of solid momentum equations, resulting in a mixture model. In fact, the phase velocities are related to each other by means of the drift factors as $u_{f}=\lambda_{u} u_{s}$ and $w_{f}=\lambda_{w} w_{s}$, whence solid phase velocity is less than fluid phase velocity if the drift factors are greater than unity, and conversely, the solid phase velocity is greater than fluid phase velocity if the drift factors are less than unity. This reveals the need of proper modeling of the drift factors in a bulk mixture mass model. Here, $\alpha_{f}\left(=1-\alpha_{s}\right)$ is the fluid volume fraction in the debris mixture, $u_{s}, w_{s}, u_{f}$ and $w_{f}$ are the solid-phase velocity and fluid-phase velocity in the down-slope and normal directions respectively. $\boldsymbol{\sigma}_{m}$ is the Cauchy stress tensor (normalized by density) and $\mathbf{f}$ is the body force (i.e., the gravity force). The Cauchy stress tensor $\boldsymbol{\sigma}_{m}$ can be written as

$$
\boldsymbol{\sigma}_{m}=-p_{m} \mathbf{I}+2 \Lambda_{\eta_{m}} \mathbf{D}_{m}
$$

where $p_{m}$ is the mixture pressure normalized by density, $\Lambda_{\eta_{m}}$ is the mixture viscosity [30]

$$
\Lambda_{\eta_{m}}=\frac{1}{2} \nu_{s}^{e} \alpha_{s}\left(\Lambda_{u}+\Lambda_{w}\right)+\frac{1}{2} \nu_{f} \alpha_{f}\left(\lambda_{u} \Lambda_{u}+\lambda_{w} \Lambda_{w}\right)
$$

where

$$
\nu_{s}^{e}=\nu_{s}+\frac{\tau_{y_{s}}}{\left\|\mathbf{D}_{m}\right\|}\left(1-e^{-m_{y}\left\|\mathbf{D}_{m}\right\|}\right)
$$

is the effective kinematic viscosity for solid [6, 43]. $\tau_{y_{s}}=\tau_{c}+\tau_{p} p_{m} / \Lambda_{p}$ is the yield stress; $\tau_{c}$ is cohesion, $\tau_{p}$ is pressure dependent parameter and $\Lambda_{p}=\alpha_{s}+\lambda_{p} \alpha_{f}$, where the drift factor for pressure, $\lambda_{p}$ relates the phase pressures by $p_{f}=\lambda_{p} p_{s}, m_{y}$ is a factor responsible for numerical purpose. $\nu_{f}$ is the kinematic viscosity for fluid. The effective viscosity that depends on drift factors, controls the dynamics of mass flow. In a simple situation, where variation of the pressure drift, $\lambda_{p}$ is negligible, the stress tensor for a viscoplastic material $\boldsymbol{\sigma}_{m}$ can be written as

$\boldsymbol{\sigma}_{m}=-p_{m} \mathbf{I}+\nu_{s} \alpha_{s} \mathbf{D}_{m}\left(\Lambda_{u}+\Lambda_{w}\right)+\nu_{f} \alpha_{f} \mathbf{D}_{m}\left(\lambda_{u} \Lambda_{u}+\lambda_{w} \Lambda_{w}\right)+\tau_{c} \nu_{D} \mathbf{D}_{m} \alpha_{s}\left(\Lambda_{u}+\Lambda_{w}\right)+\tau_{p} p_{m} \nu_{D} \alpha_{s} \mathbf{D}_{m}\left(\Lambda_{u}+\Lambda_{w}\right) / \Lambda_{p}$,

where $\tau_{c}$ is cohesion, $\nu_{D}=\frac{1-e^{-m_{y}\left\|\mathbf{D}_{m}\right\|}}{\left\|\mathbf{D}_{m}\right\|}, \mathbf{D}_{m}=\frac{1}{2}\left[\nabla \mathbf{u}_{m}^{\Lambda}+\left(\nabla \mathbf{u}_{m}^{\Lambda}\right)^{T}\right]$ is the (deformation) strain-rate tensor. If $\lambda_{u}, \lambda_{w}, \lambda_{p}$ are close to 1 , then $u_{s}=u_{m}, w_{s}=w_{m}$ and $p_{s}=p_{m}$ as often used in single-phase granular and debris flows [5, 6] for which $\Lambda_{\eta_{m}}=\Lambda_{m}=\nu_{s}^{e} \alpha_{s}+\nu_{f} \alpha_{f}$. The generalized quasi two-phase mixture model [30] consists of the following non-linear PDEs:

$$
\begin{aligned}
& \frac{\partial u_{m}}{\partial x}+\frac{\partial w_{m}}{\partial z}=0 \\
& \begin{aligned}
\frac{\partial u_{m}}{\partial t}+\frac{\partial}{\partial x}\left(\Lambda_{u u} u_{m}^{2}\right)+\frac{\partial}{\partial z}\left(\Lambda_{u w} u_{m} w_{m}\right) & =f_{x}-\frac{\partial p_{m}}{\partial x} \\
+ & 2 \frac{\partial}{\partial x}\left[\Lambda_{\eta_{u}} \frac{\partial\left(\Lambda_{u} u_{m}\right)}{\partial x}\right]+\frac{\partial}{\partial z}\left[\Lambda_{\eta_{u}} \frac{\partial\left(\Lambda_{u} u_{m}\right)}{\partial z}+\Lambda_{\eta_{w}} \frac{\partial\left(\Lambda_{w} w_{m}\right)}{\partial x}\right] \\
\frac{\partial w_{m}}{\partial t}+\frac{\partial}{\partial x}\left(\Lambda_{u w} u_{m} w_{m}\right)+\frac{\partial}{\partial z}\left(\Lambda_{w w} w_{m}^{2}\right) & =f_{z}-\frac{\partial p_{m}}{\partial z} \\
+ & \frac{\partial}{\partial x}\left[\Lambda_{\eta_{u}} \frac{\partial\left(\Lambda_{u} u_{m}\right)}{\partial z}+\Lambda_{\eta_{w}} \frac{\partial\left(\Lambda_{w} w_{m}\right)}{\partial x}\right]+2 \frac{\partial}{\partial z}\left[\Lambda_{\eta_{w}} \frac{\partial\left(\Lambda_{w} w_{m}\right)}{\partial z}\right]
\end{aligned}
\end{aligned}
$$

where $u_{m}=\left(\alpha_{s}+\lambda_{u} \alpha_{f}\right) u_{s}$ and $w_{m}=\left(\alpha_{s}+\lambda_{w} \alpha_{f}\right) w_{s}$ are the mixture velocities in $x$ - and $z$-directions respectively, $p_{m}=\left(\alpha_{s}+\lambda_{p} \alpha_{f}\right) p_{s}$ is the mixture pressure and $\left(f_{x}, f_{z}\right)$ are the components of the body force f. The inertial and dynamical coefficients that appear in momentum balance equations (7) and (8) are

$$
\begin{aligned}
& \Lambda_{u u}=\frac{\alpha_{s}+\lambda_{u}^{2} \alpha_{f}}{\left(\alpha_{s}+\lambda_{u} \alpha_{f}\right)^{2}}, \Lambda_{w w}=\frac{\alpha_{s}+\lambda_{w}^{2} \alpha_{f}}{\left(\alpha_{s}+\lambda_{w} \alpha_{f}\right)^{2}}, \Lambda_{u w}=\frac{\alpha_{s}+\lambda_{u} \lambda_{w} \alpha_{f}}{\left(\alpha_{s}+\lambda_{u} \alpha_{f}\right)\left(\alpha_{s}+\lambda_{w} \alpha_{f}\right)}, \Lambda_{u}=\frac{1}{\alpha_{s}+\lambda_{u} \alpha_{f}}, \\
& \Lambda_{w}=\frac{1}{\alpha_{s}+\lambda_{w} \alpha_{f}}, \Lambda_{\eta_{u}}=\nu_{s} \alpha_{s}+\lambda_{u} \nu_{f} \alpha_{f}, \Lambda_{\eta_{w}}=\nu_{s} \alpha_{s}+\lambda_{w} \nu_{f} \alpha_{f}, \Lambda_{p}=\alpha_{s}+\lambda_{p} \alpha_{f} .
\end{aligned}
$$


The following pressure-Poisson equation (10) results from the use of the momentum equations (7), (8) and the equation of continuity, [6] [30] and makes the system closed so that the numerical integration possible.

$$
\frac{\partial^{2} p_{m}}{\partial x^{2}}+\frac{\partial^{2} p_{m}}{\partial z^{2}}=\frac{\partial F}{\partial x}+\frac{\partial G}{\partial z}
$$

where

$$
\begin{aligned}
& F=-\frac{\partial}{\partial x}\left(\Lambda_{u u} u_{m}^{2}\right)-\frac{\partial}{\partial z}\left(\Lambda_{u w} u_{m} w_{m}\right)+2 \frac{\partial}{\partial x}\left[\Lambda_{\eta_{u}} \frac{\partial\left(\Lambda_{u} u_{m}\right)}{\partial x}\right]+\frac{\partial}{\partial z}\left[\Lambda_{\eta_{u}} \frac{\partial\left(\Lambda_{u} u_{m}\right)}{\partial z}+\Lambda_{\eta_{w}} \frac{\partial\left(\Lambda_{w} w_{m}\right)}{\partial x}\right]+f_{x} \\
& G=-\frac{\partial}{\partial x}\left(\Lambda_{u w} u_{m} w_{m}\right)-\frac{\partial}{\partial z}\left(\Lambda_{w w} w_{m}^{2}\right)+\frac{\partial}{\partial x}\left[\Lambda_{\eta_{u}} \frac{\partial\left(\Lambda_{u} u_{m}\right)}{\partial z}+\Lambda_{\eta_{w}} \frac{\partial\left(\Lambda_{w} w_{m}\right)}{\partial x}\right]+2 \frac{\partial}{\partial z}\left[\Lambda_{\eta_{w}} \frac{\partial\left(\Lambda_{w} w_{m}\right)}{\partial z}\right]+f_{z}
\end{aligned}
$$

The model equations (7)-10 are in conservative form with three unknowns, namely the mixture velocities, $u_{m}, w_{m}$, and the pressure $p_{m}$. For the detailed discussion of the generalized quasi two-phase bulk mixture model, the reduced forms- (i) pressure drift close to unity, (ii) identical velocity drifts factors, (iii) velocity drifts factors close to unity, and the essence of the model, one can refer to Pokhrel et al. [30.

\section{Results and Discussions}

In order to study the behaviour of inertial and dynamical coefficients, slip velocity, front position of the debris mixture and effective viscosity of a bulk mixture mass released from a silo gate that moves down a chute, we integrate the model equations $77-10$ numerically. The initial set-up is as shown in Fig. 1 . The channel inclination angle is $\zeta=45^{\circ}$. The debris material enters into the channel at $x=0$ with an inlet height $h_{\text {in }}=0.15 \mathrm{~m}$ and an average inlet velocity of $u_{m_{\text {in }}}=0.9 \mathrm{~ms}^{-1}$. A full dimensional debris bulk mixture simulations in complex three-dimensional flow structures has been presented by von Boetticher et al. 43. They used the volume-of-fluid ( VoF) approach to combine the mixed phase and the air phase into a single cell-averaged Navier-Stokes equations for incompressible flow with the open source CFD software,

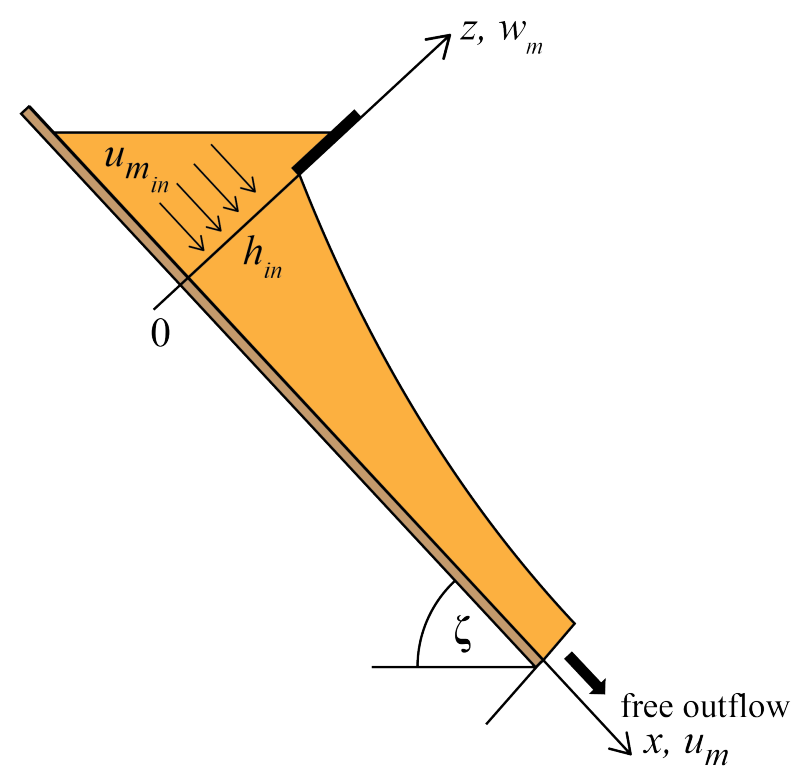

Figure 1: Side view of the inclined channel used for the simulation of flow of debris mixture material out of the silo inlet with subjected to a free outflow. The silo inlet has the opening $h_{\text {in }}$ and the inlet velocity is $u_{m_{i n}} . \quad \zeta$ is the channel inclination angle, $u_{m}$ and $w_{m}$ denote the mixture velocities in the downslope ( $x$-directon) and in the slope normal ( $z$-direction) respectively. (Adopted from Domnik and Pudasaini [5]). 


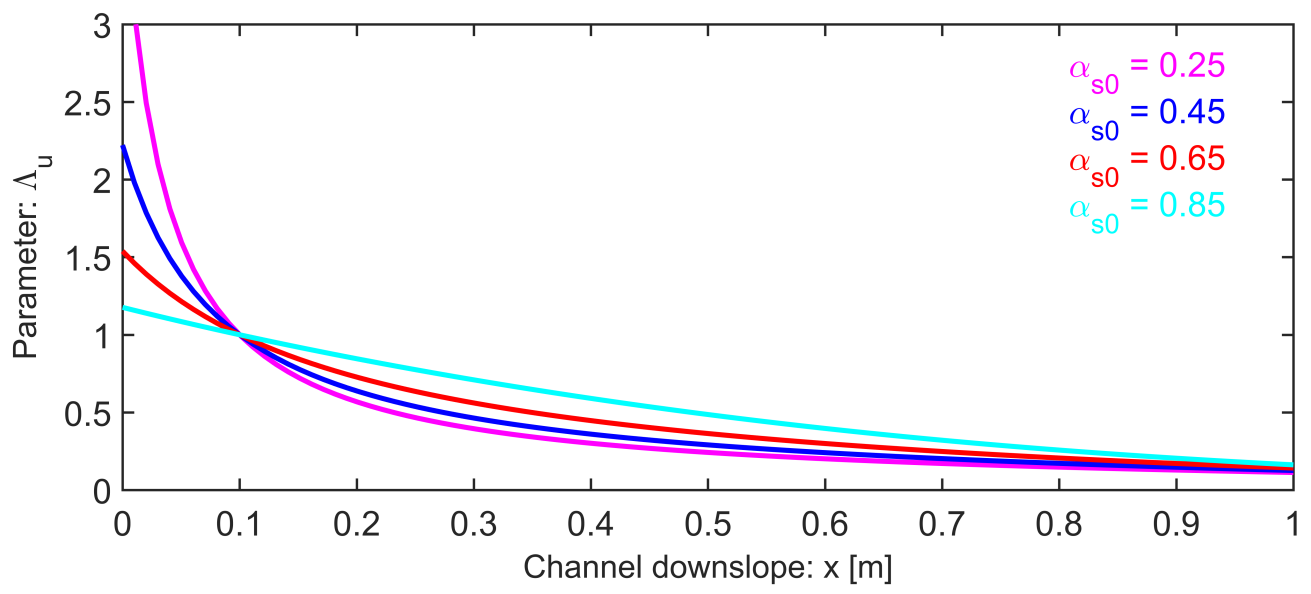

Figure 2: The behaviour of $\Lambda_{u}$ in downslope distance.
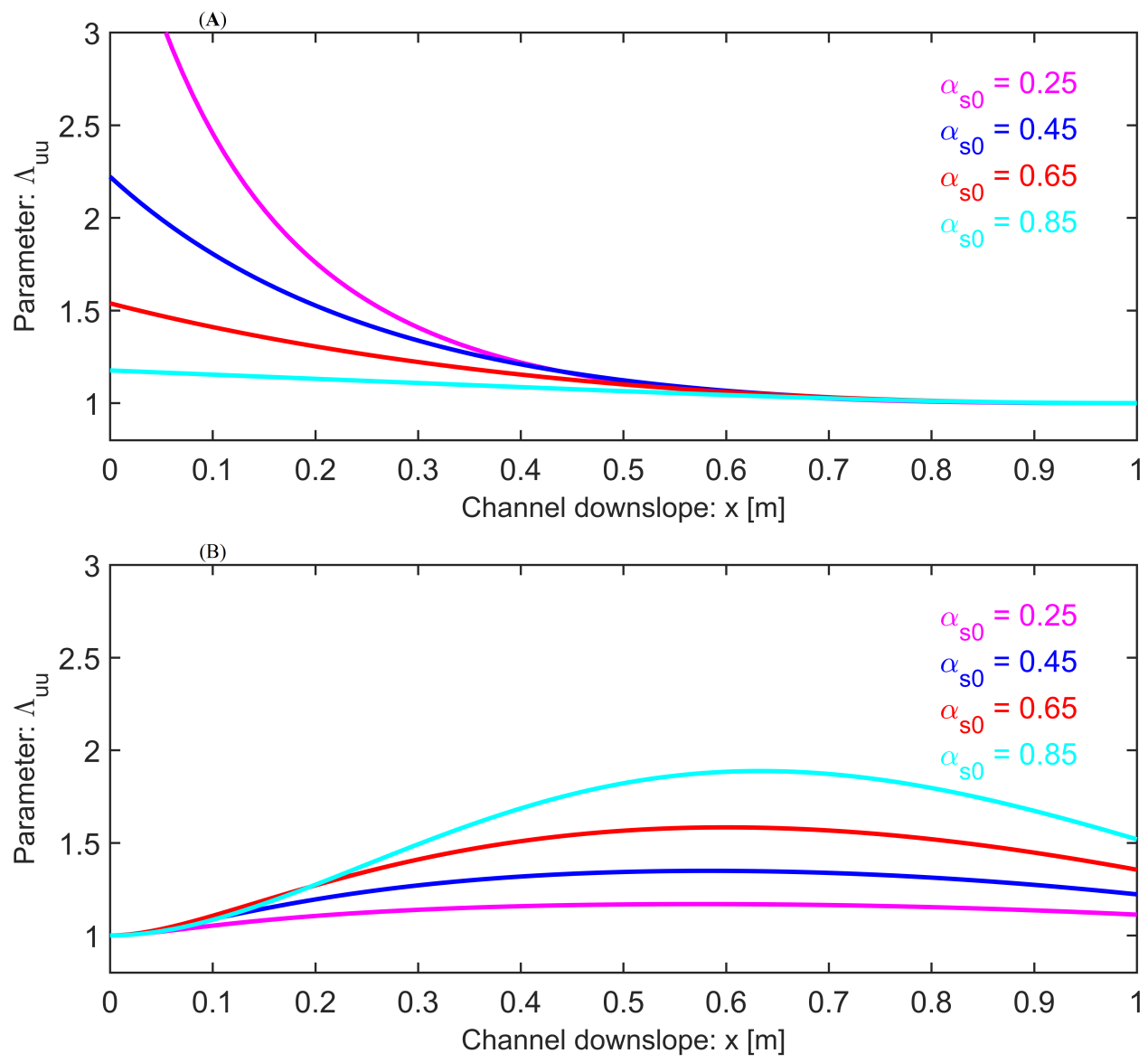

Figure 3: Evolution of inertial dynamical coefficient $\Lambda_{u u}$ along the channel for different initial solid-volume fractions $\alpha_{s_{0}}=0.25,0.45,0.65$ and $0.85(\mathrm{~A})$ for $\lambda_{u}, \lambda_{w}<1$ and (B) for $\lambda_{u}, \lambda_{w}>1$. The parameter $\Lambda_{u u}$ depends on solid volume fraction and drift factors.

OpenFOAM. But, our numerical method is based on the extended NaSt2D [5, 6, 9], an extended computer 

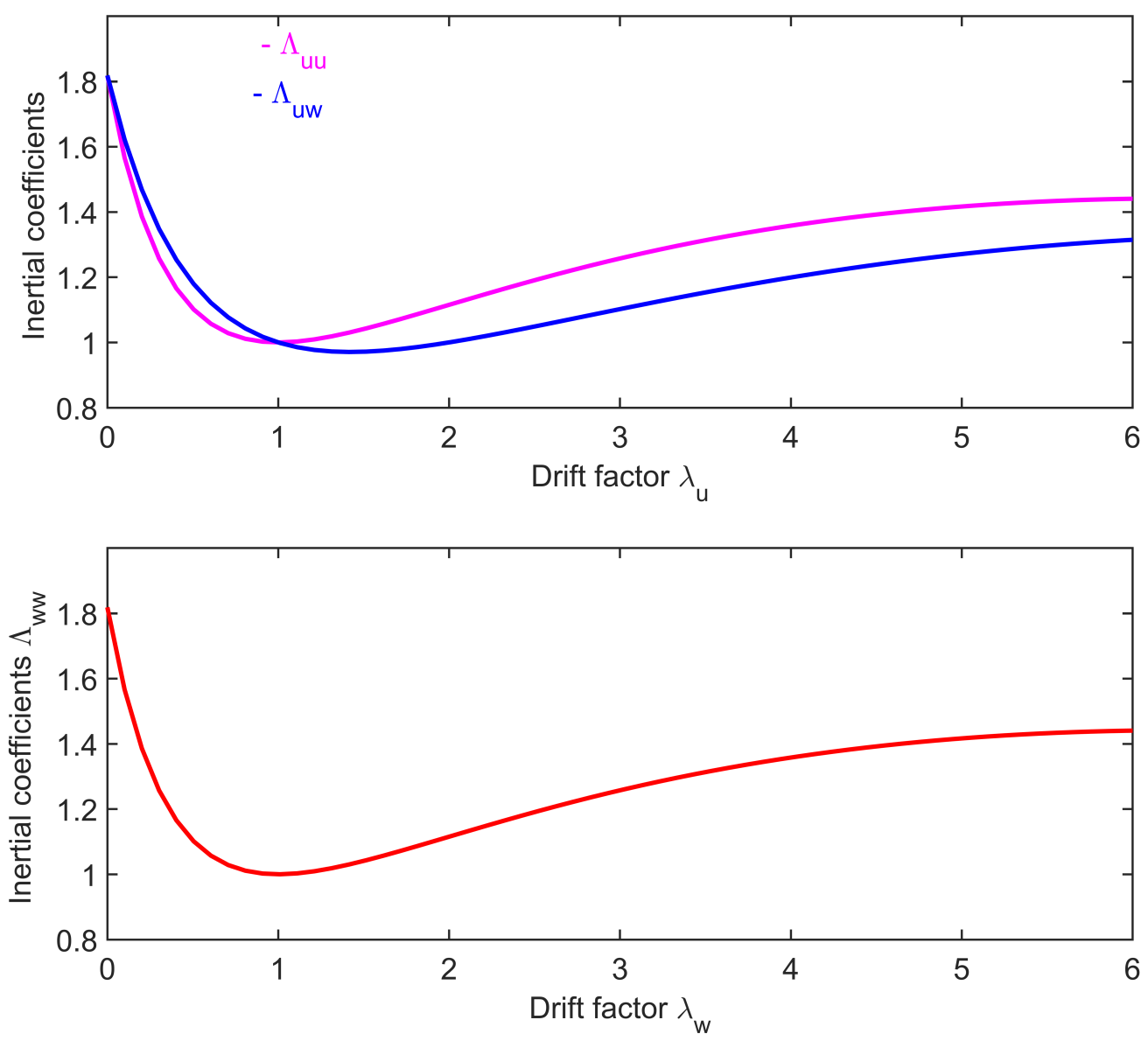

Figure 4: Variation of the inertial coefficients $\Lambda_{u u}, \Lambda_{u w}$ (top panel) and $\Lambda_{w w}$ (bottom panel) for the same initial solid volume fraction $\alpha_{s_{0}}=0.65$ with the downslope velocity drift, $\lambda_{u}$.

code using the finite-volume method for the simulation of incompressible non-Newtonian fluids and complex granular mixtures. In both the models of Domnik and Pudasaini [5] and von Boetticher et al. [43], pressureand rate-dependent Coulomb-viscoplastic slip conditions [6] are implemented as bottom boundary condition that dynamically evolve during the flow.

Figure 2 plots the variation of the relative downslope velocity for solid with respect to the mixture velocity, $\Lambda_{u}$ with the downslope distance for different initial solid volume fractions $\alpha_{s_{0}}=0.25,0.45,0.65$ and 0.85 . $\Lambda_{u}$ shows non-linear behaviour for each $\alpha_{s}$. The value for $\Lambda_{u}$ is higher for dilute and is lower for dense mixture before the flow exits the silo. The values for $\Lambda_{u}$ begin to decrease as the flow moves downslope. The rate of decrease is higher for more dilute flows such that at the silo gate $(x=0.1 \mathrm{~m}), \Lambda_{u}$ for different $\alpha_{s}$ coincide. This decrease of all $\Lambda_{u}$ continues as the flow moves downslope. But after the silo gate, the values for $\Lambda_{u}$ become greater for dense flow. At the right end of the channel $(x=1 \mathrm{~m})$, they nearly coincide. In fact, $\Lambda_{u}$ depends on $\alpha_{s}$, and $\alpha_{s}$ is a state variable, which in turn, depends on $x$ as given by advection-diffusion equation presented in Iverson and Denlinger [15, Pudasaini et al. [37, Khattri 23] and Pokhrel et al. 30. The analytical solution of $\alpha_{s}$ from the advection-diffusion equation can be found in Khattri 23]. Figure 3 represents the evolution of the inertial coefficient $\Lambda_{u u}$ along the downslope distance for different initial solid volume fractions. Figure $3 \mathrm{~A}$ is with drift factors $\lambda_{u}, \lambda_{w}<1$ and Fig. $3 \mathrm{~B}$ is that for $\lambda_{u}, \lambda_{w}>1$. In Fig. 3A A, the inertial coefficient $\Lambda_{u u}$ takes up higher values for more dilute flows. As the flow is dense, $\Lambda_{u u}$ decreases. For different $\alpha_{s}, \Lambda_{u u}$ evolve such that they decrease as the mixture moves downslope so as to coincide asymptotically. Thus, $\Lambda_{u u}$ highly depends upon $\alpha_{s}$ for early stage of evolution. 


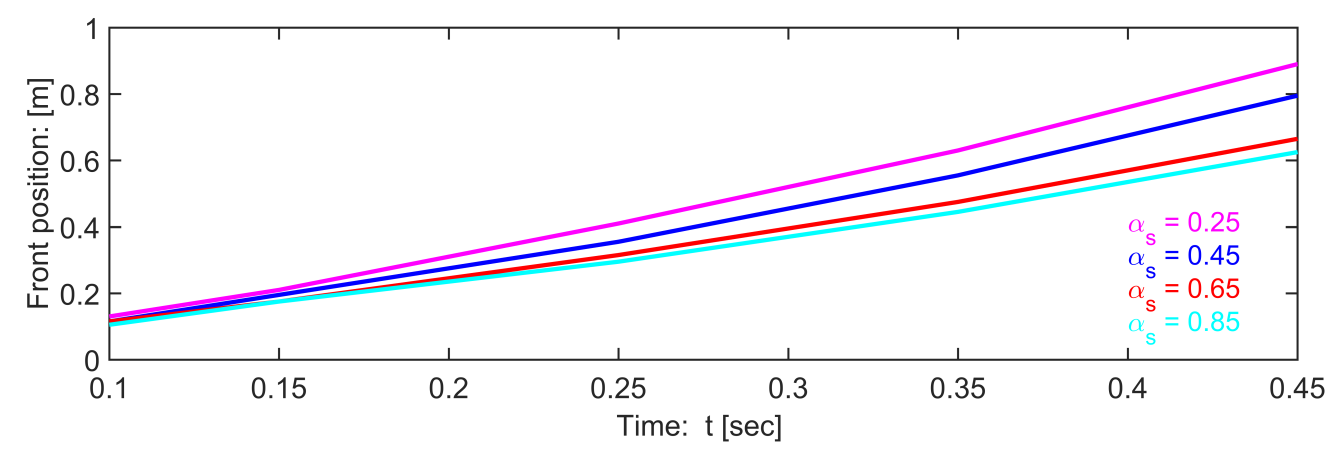

Figure 5: Time evolution of the front position of the debris for different solid volume fractions, $\alpha_{s}=$ $0.25,0.45,0.65$ and 0.85 .

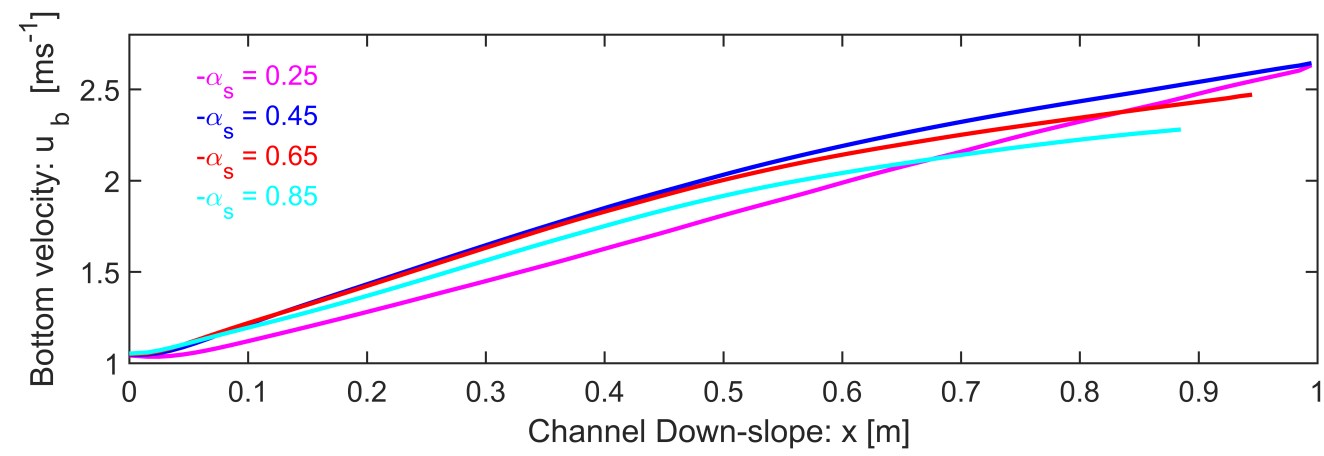

Figure 6: Bottom slip velocity along the channel for different solid volume fractions $\alpha_{s}=0.25,0.45,0.65$ and 0.85 at time $t=0.45 \mathrm{~s}$.

But later, as the flow is more dispersed, it is more independent of $\alpha_{s}$. Fig. $3 \mathrm{~B}$ shows completely different behaviour of $\Lambda_{u u}$ for drift factors greater than unity: it increases more for dense flows in downslope. $\Lambda_{u w}$ and $\Lambda_{w w}$ show similar behaviour as the influencing factors are $\lambda_{u}, \lambda_{w}, \alpha_{s}$. This is the reason why we do not present them here.

However, Fig. 4 makes a comparison of the different inertial coefficients $\Lambda_{u u}, \Lambda_{u w}$ and $\Lambda_{w w}$ for the same initial material composition, $\alpha_{s_{0}}=0.65$ against the downslope velocity drift, $\lambda_{u}$ (top) and $\lambda_{w}$ (bottom). They evolve differently and show similar non-linear behaviour. For the drift factor $\lambda_{u}$ less than unity, $\Lambda_{u w}$ dominates the inertial coefficient $\Lambda_{u u}$, whereas for $\lambda_{u}$ greater than unity, $\Lambda_{u u}$ dominates the $\Lambda_{u w}$. For $\lambda_{u}<1$, all the inertial coefficients quickly decrease, acquire their minima in between the transition $1<\lambda_{u}<2$, and slowly increase thereafter.

Figure 5 plots the evolution of the front position of the debris for different values of the initial solid volume fractions, $\alpha_{s}=0.25,0.45,0.65$ and 0.85. For all $\alpha_{s}$, the front positions shift farther downslope as time progresses. At each time slice, the front position is at more downslope for more dilute flows, due to the reduced mixture viscosity. The time evolution of the front position of the mixture is qualitatively similar to that presented in von Boetticher et al. 44. The detailed analysis of the front position has been presented in Khattri and Pudasaini [24].

Fig. 6 provides important information about the slip velocity at the bottom of the channel that is set to zero in classical Newtonian fluid simulation. In real flow situation, this is not valid. Here, the slip velocity along the channel is shown for $t=0.45 \mathrm{~s}$ for different solid volume fractions. The results show that the slip velocity increases along the slope and generally it attains higher values for lower values of $\alpha_{s}$ except for $\alpha_{s}=0.25$, which behaves a bit differently than others. The reason for this is the viscous fluid dominance. 
Some Simulation Results and Parameter Analyses of a Generalized Quasi Two-Phase Bulk Mixture Model

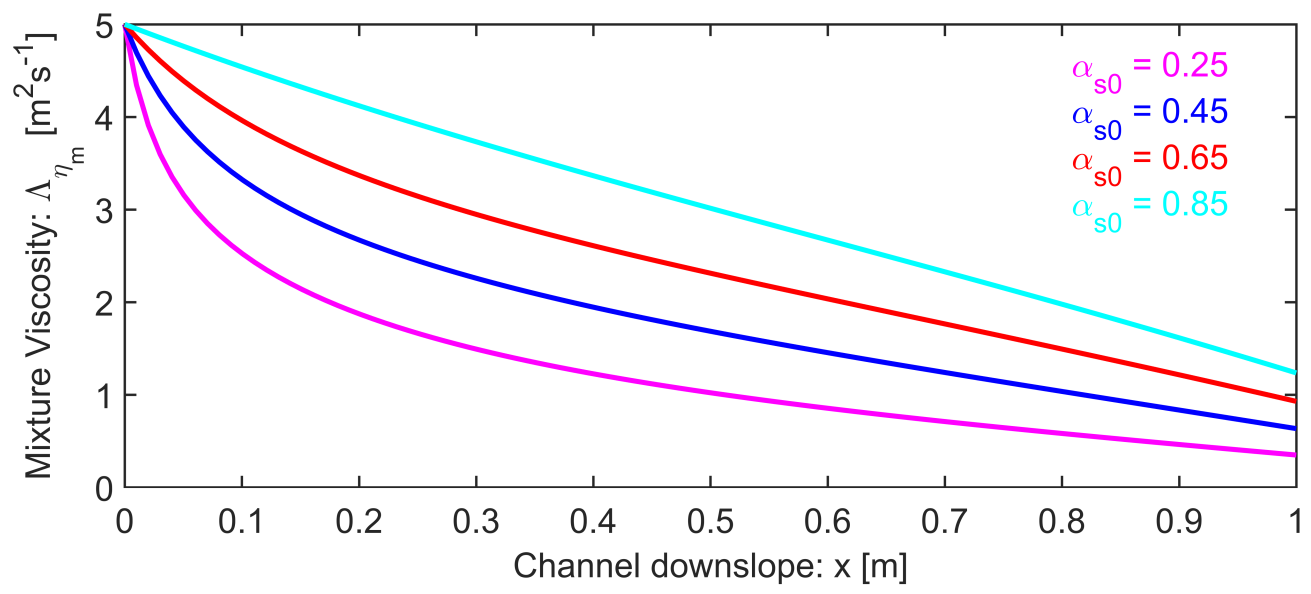

Figure 7: Variation of the generalized mixture viscosity along the channel that depends strongly on the initial material composition of solid volume fraction and shows a shear thinning behaviour.

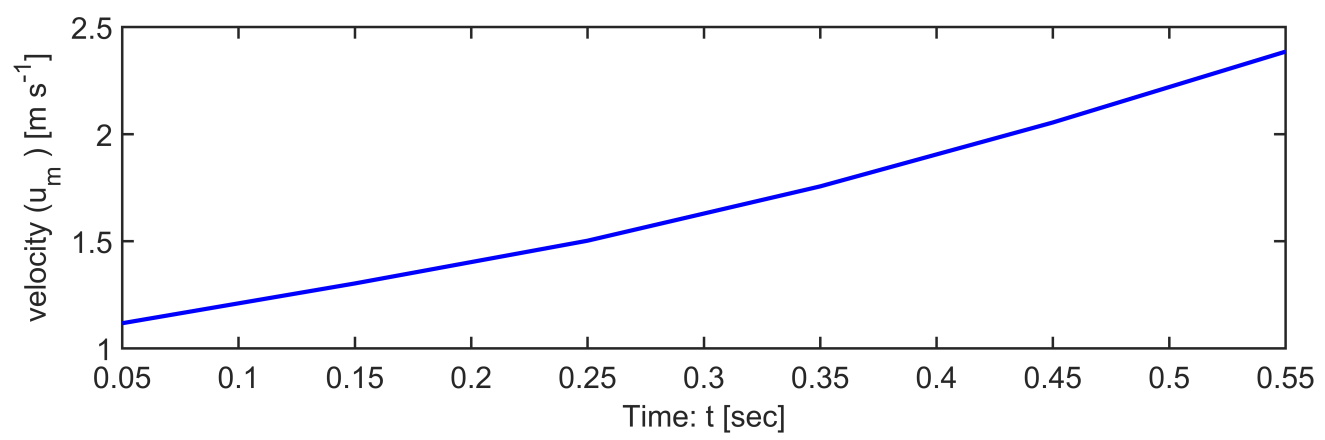

Figure 8: Time evolution of the maximum velocity for $\alpha_{s}=0.65$.

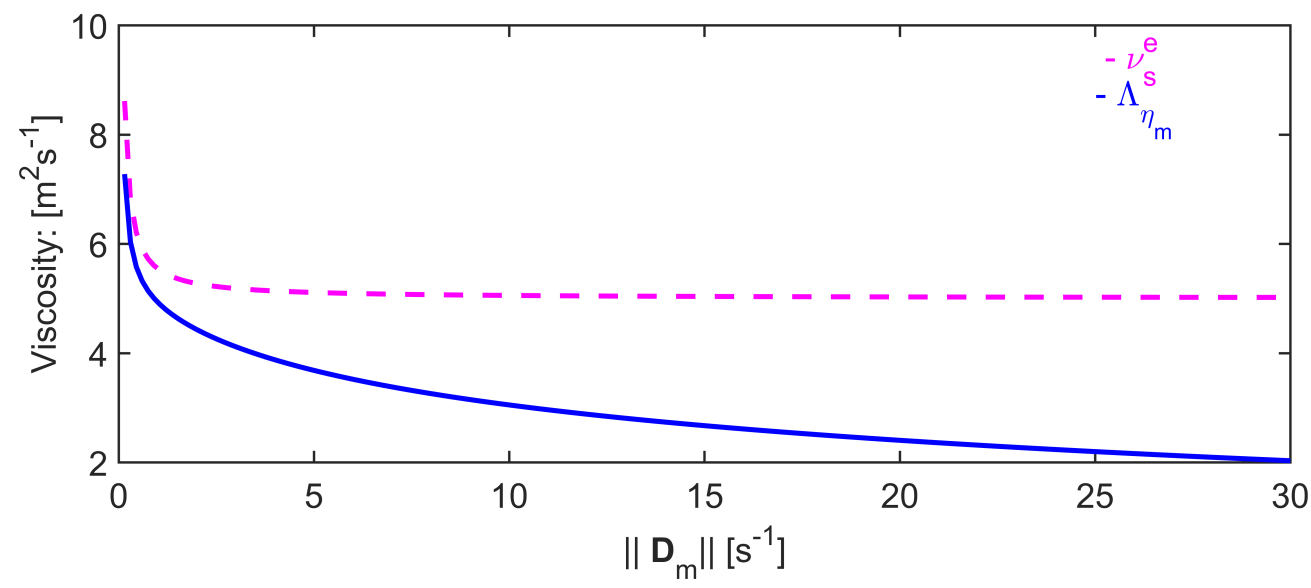

Figure 9: Comparison of the mixture viscosity (3) and effective viscosity (4) versus norm of strain rate tensor. 
Figure 7 shows the spatial evolution of the mixture viscosity, $\Lambda_{\eta_{m}}$ for different values of the solid volume fractions, $\alpha_{s}=0.25,0.45,0.65$ and 0.85 . Each $\Lambda_{\eta_{m}}$ shows non-linear behaviour except for $\alpha_{s}=0.85$, where the flow is relatively dense. Each $\Lambda_{\eta_{m}}$ decreases as flow moves downslope due to the dispersion of the flow as described by the pressure-rate dependent rheology.

Figure 8 presents the time evolution of the maximum velocity of the debris mixture. The maximum velocity increases due to the accelerated mixture flow down a slope and reaches a downslope velocity of $2.4 \mathrm{~ms}^{-1}$ at $t=0.55 \mathrm{~s}$.

Figure 9 plots the comparison of the mixture viscosity (3) (Pokhrel et al., 2018) and the effective viscosity (4) (Domnik et al., 2013) versus norm of strain rate tensor, $\left\|\mathbf{D}_{m}\right\|$. The chosen parameters are $\nu_{s}=5$ $\mathrm{m}^{2} / \mathrm{s}, \nu_{f}=10^{-3} \mathrm{~m}^{2} / \mathrm{s}, \tau_{p}=0.5446$ and $\alpha_{s}=0.65$. As $\left\|\mathbf{D}_{m}\right\|$ increases, both $\nu_{s}^{e}$ and $\Lambda_{\eta_{m}}$ both decrease sharply. $\Lambda_{\eta_{m}}$ continues decreasing but $\nu_{s}^{e}$ remains almost constant.

\section{Conclusions}

Here, we analyzed the behaviour of some inertial and dynamical coefficients of the generalized quasi twophase bulk mixture model [30] and simulated the evolution of some dynamical variables. The model is a set of coupled partial differential equations with new mechanical and dynamical aspects of effective friction, generalized bulk and shear viscosities, mixture velocities and pressure. These variables are functions of several evolving inertial and dynamical coefficients, parameters and drift factors. The spatial evolutions of the parameters $\Lambda_{u}, \Lambda_{u u}$ and $\Lambda_{u w}$ show interesting non-linear behaviour. The behaviour of $\Lambda_{u}$ is different for different solid volume fractions for the mixture flow before and after silo inlet. $\Lambda_{u u}, \Lambda_{u w}$, and $\Lambda_{w w}$ also show different non-linear behaviours for the same material composition and different values of the drift factor $\lambda_{u}$. They show a sharp decrease for $0<\lambda_{u}<1$ and increase relatively gently for $\lambda_{u}>1$. As a simulation output, we also studied the behaviour of the front position of the debris material. The front moves more downslope for dilute flows and less downslope for dense flows due to different mixture viscosities. We also analyzed the bottom slip velocities for varying material compositions and observed that they also behave differently for the early stage of evolution and later. Generalized mixture viscosity decreases as the flow moves downslope and shows more non-linear behaviour for increasing initial solid volume fractions. Simulation result show that the maximum velocity of the debris body increases as the time progresses. We also compared the effective viscosity as defined in Domnik et al. [6] and the bulk mixture viscosity as constructed in Pokhrel et al. [30] with respect to the norm of the strain rate tensors. For the increasing norm of strain-rate tensor, the effective viscosity shows almost linear and constant behaviour where as the bulk mixture viscosity varies strongly non-linearly. These results may help in the choice of parameters for the model simulations of mixture mass flows.

\section{Acknowledgments}

We thank the reviewers, and the Executive Editor Prof. Dr. Dil B. Gurung for their constructive reviews and suggestions that resulted in the substantially improved paper. Parameshwari Kattel acknowledges University Grants Commission (UGC), Nepal for the financial support provided as a PhD fellowship (PhD2071/072-Sci. \& Tech.-03).

\section{References}

[1] Arai, M. and Takahashi, T. (1986) The Karman constant of the flow laden with high sediment. Proc. of the 3rd International Symposium on River Sedimentation, University of Missisipi, 824-833.

[2] Bagnold, R. A. (1954) Experiments on a gravity-free dispersion of large solid spheres in a Newtonian fluid under shear. Proc. R. Soc. Lond., Ser. A, 225, 49-63.

[3] Chen, C. L. (1988) Generalized viscoplastic modeling of debris flow. J. Hydraul. Res., 114(3), $237-258$. 
$\underline{\text { Some Simulation Results and Parameter Analyses of a Generalized Quasi Two-Phase Bulk Mixture Model }}$

[4] Dent, J. D. and Lang, T. E. (1983) A biviscous modified Binghman model of snow avalanche motion. Annals of Glaciology, 4, 42-46.

[5] Domnik, B. and Pudasaini, S. P. (2012) Full two-dimensional rapid chute flows of simple viscoplastic granular materials with a pressure-dependent dynamic slip-velocity and their numerical simulations. J. Non-Newtonian Fluid Mech., 173-174, 72-86.

[6] Domnik, B., Pudasaini, S. P., Katzenbach, R. and Miller, S. A. (2013) Coupling of full two-dimensional and depth-averaged models for granular flows. J. Non-Newtonian Fluid Mechanics, 201, 56-68.

[7] Fernandez-Nieto, E. D., Bouchut, F., Bresch, D., Daz, M. J. C. and Mangeney, A. (2008) A new Savage-Hutter type model for submarine avalanches and generated tsunami. J. Comput. Phys., 227(16), $7720-7754$.

[8] Gray, J. M. N. T., Wieland, M. and Hutter, K. (1999) Gravity-driven free surface flow of granular avalanches over complex basal topography. Proc. R. Soc. Lond., 455, 1841-1874.

[9] Griebel, M., Dornseiferand, T. and Neunhoeffer, T. (1997) Numerical Simulation in Fluid Dynamics: A Practical Introduction. Society for Industrial and Applied Mathematics, Philadelphia.

[10] Guo, S., Xu, P., Zheng, Z. and Gao, Y. (2015) Estimation of flow velocity for a debris flow via the two-phase fluid model. Nonlin. Processes Geophys., 22, 109-116.

[11] Guthrie, R., Mitchell, J., Lanquaye-Opoku, N. and Evans, S. (2010) Extreme weather and landslide initiation in coastal British Columbia. J. Eng. Geol. Hydroge., 43, 417-428, 6350.

[12] Hungr, O. (1995) A model for the runout analysis of rapid flow slides, debris flows and avalanches. Can. Geotech. J., 32(4), 610-623.

[13] Hutter, K., Svendsen, B. and Rickenmann, D. (1996) Debris flow modeling review. Continuum Mech. Thermo. dyn., 8, 1-35.

[14] Iverson, R. M. 1997, The physics of debris flows. Rev. Geophys., 35(3), 245-296.

[15] Iverson, R. M. and Denlinger, R. P. (2001) Flow of variably fluidized granular masses across threedimensional terrain: 1. Coulomb mixture theory. J. Geophys. Res., 106(B1), 537-552.

[16] Jan, C. D. (1997) A study on the numerical modeling of debris flow. Debris Flow Hazard Mitigation: Mechanics, Prediction and Assessment, Eds. Chen, New York, 717-726.

[17] Johnson, A. M. (1970) Physical Processes in Geology. Freeman, San Francisco.

[18] Kafle, J., Pokhrel, P. R., Khattri, K. B., Kattel, P., Tuladhar, B. M. and Pudasaini, S. P. (2016) Submarine landslide and particle transport in mountain lakes, reservoirs and hydraulic plants. Annals of Glaciology, 57(71) doi: 10.3189/2016AoG71A034.

[19] Kafle, J. and Tuladhar, B. M. (2018) Landslide-water Interaction for Partially Submerged Landslide. Journal of Nepal Mathematical Society, 1(1) 22-29.

[20] Kattel, P., Khattri, K. B., Pokhrel, P. R., Kafle, J., Tuladhar, B. M. and Pudasaini, S. P. (2016) Simulating Glacial lake outburst floods with a two-phase mass flow model. Annals of Glaciology, 57(71) (doi: 10.3189/2016AoG71A039).

[21] Kattel, P., Kafle, J., Fischer, J.-T., Mergili, M., Tuladhar, B. M. and Pudasaini, S. P. (2018) Interaction of two-phase debris flow with obstacles. Eng. Geol., 242, 197-217 (doi: 10.1016/j.enggeo.2018.05.023).

[22] Kattel, P. and Tuladhar, B. M. (2018) Interaction of two-phase debris flow with lateral converging shear walls. Journal of Nepal Mathematical Society, 1(2), 40-52.

[23] Khattri, K. B. (2014) Sub-diffusive and Sub-advective Viscous Fluid Flows in Debris and Porous Media. M. Phil. Dissertation, Kathmandu University, Kavre, Dhulikhel, Nepal 
[24] Khattri, K. B. and Pudasaini, S. P. (2018) An extended quasi two-phase mass flow model. Int. J. Non-Linear Mech., 106, 205-222. (doi: 10.1016/j.ijnonlinmec.2018.07.008).

[25] Khattri, K. B., Fischer, J.-T., Jaboyedoff, M. and Pudasaini, S. P. (2018) Wet snow avalanche simulations to assess flow-obstacle-interactions and potential defense structure designs. Proceeding, International Snow Science Workshop, Innsbruck, Austria, 711-715.

[26] Khattri, K. B. and Pudasaini, S. P. (2019) Channel flow simulation of a mixture with a full-dimensional generalized quasi two-phase model Mathematics and Computers in Simulation. doi:10.1016/j.matcom.2019.03.014

[27] Norem, H., Locat, J. and Schieldrop, B. (1990), An approach to the physics and the modeling of the submarine flow slides. Marine Geotechnical, 9, 93-111.

[28] O'Brien, J. S., Julien, P. Y. and Fullerton, W. T. (1993) Two-dimensional water flood and mud flow simulation. J. Hyd. Eng., 119(2), 244-261.

[29] Pitman, E. B. and Le, L. (2005) A two fluid model for avalanche and debris flows. Phil. Trans. R. Soc. A, 363(3), 1573-1601.

[30] Pokhrel, P. R., Khattri, K. B., Tuladhar, B. M. and Pudasaini, S. P. (2018) A generalized quasi twophase bulk mixture model for mass flow. International Journal of Non-Linear Mechanics, 99, 229-239.

[31] Prochaska, A. B., Santi, P. M., Higgins, J. D. and Cannon, S. H. (2008) A study of methods to estimate debris flow velocity. Landslides, 5, 431-444.

[32] Pudasaini, S. P. (2011) Some exact solutions for debris and avalanche flows. Phys. Fluids, 23(4), 043301.

[33] Pudasaini, S. P. (2012) A general two-phase debris flow model. J. Geophysics. Res., 117, F03010 (doi: 10.1029/2011JF002186).

[34] Pudasaini, S.P. and Hutter, K. (2003) Rapid shear flows of dry granular masses down curved and twisted channels. J. Fluid Mech., 495, 193-208.

[35] Pudasaini, S. P. and Hutter, K. (2007) Avalanche Dynamics: Dynamics of Rapid Flows of Dense Granular Avalanches. Springer, Berlin, New York.

[36] Pudasaini, S. P., Wang, Y. and Hutter, K. (2005) Modelling debris flows down general channels. Nat. Hazards Earth Syst. Sci., 5, 799-819.

[37] Pudasaini, S. P., Ghosh Hajra, S., Kandel, S. and Khattri, K. B. (2018) Analytical solutions to a non-linear diffusion-advection equation. Z. Angew. Math. Phys., 69(6) 150.

[38] Rickenmann, D., Laigle, D., McArdell, B. W. and Hubl, J. (2006) Comparison of 2D debris-flow simulation models with field events. Computational Geosciences, 10, 241-264.

[39] Revellino, P., Hungr, O., Guadagno, F. M. and Evans, S. G. (2004) Velocity and runout simulation of destructive debris flows and debris avalanches in pyroclastic deposits. Campania region, Italy, Environ. Geol., 45(3), 295-311.

[40] Savage, S. B. and Hutter, K. (1989) The motion of a finite mass of granular material down a rough incline. J. Fluid Mech., 199, 177-215.

[41] Takahashi, T. (1981) Debris flow. Ann. Rev. Fluid Mech., 13, 57-77.

[42] Takahashi, T. (2007) Debris Flow: Mechanics, Prediction and Countermeasures. Taylor and Francis, New York. 
Some Simulation Results and Parameter Analyses of a Generalized Quasi Two-Phase Bulk Mixture Model

[43] von Boetticher, A., Turowski, J. M., McArdell, B. W., Rickenmann, D. and Kirchner, J. W. (2016) DebrisInterMixing-2.3: a finite volume solver for three-dimensional debris-flow simulations with two calibration parameters - Part 1: Model description. Geosci. Model Dev., 9, 2909-2923 (doi: 10.5194/gmd-9-2909-2016).

[44] von Boetticher, A., Turowski, J. M., McArdell, B. W., Rickenmann, D., Hürlimann, M., Scheidl, C. and Kirchner, J. W. (2017) DebrisInterMixing-2.3: A finite volume solver for three-dimensional debris - flow simulations with two calibration parameters - Part 2: Model validation. Geosci. Model Dev. Discuss., 10, 3963-3978 (doi:10.5194/gmd-10-3963-2017).

[45] Worni, R., Huggel, C., Clague, J. J., Schaub, Y. and Stoffel, M. (2014) Coupling glacial lake impact, dam breach, and flood processes: a modeling perspective. Geomorphology, 224, 161-176.

[46] Zahibo, N., Pelinovsky, E., Talipova, T. and Nikolkina, I. (2010) Savage-Hutter model for avalanche dynamics in inclined channels: Analytical solutions, J. Geophys. Res., 115, B03402, (doi:10.1029/2009JB006515). 